\title{
Comparação entre o Método Trecho-a-Trecho e o Método de Múltiplas Saídas no dimensionamento de uma linha lateral de um sistema de irrigação por aspersão
}

\section{Eugenio Paceli de Miranda ${ }^{1}$, Gilbenes Bezerra Rosal ${ }^{2}$}

${ }^{1}$ Instituto Federal de Educação, Ciência e Tecnologia do Ceará - IFCE. Campus Iguatu. Rodovia Iguatu-Várzea Alegre, km 05, S/No. Vila Cajazeiras. Iguatu-CE, Brasil (CEP 63500-000).E-mail: eu.paceli@yahoo.com.br.

${ }^{2}$ Curso de Graduação em Tecnologia em Irrigação e Drenagem. Instituto Federal de Educação, Ciência e Tecnologia do Ceará - IFCE. Campus Iguatu. Rodovia IguatuVárzea Alegre, km 05, S/Nº. Vila Cajazeiras. Iguatu-CE, Brasil (CEP 63500-000).

Resumo. O dimensionamento de sistemas de irrigação é imprescindível para o seu bom funcionamento e tem uma relação direta com o consumo de energia elétrica pelo conjunto motobomba. 0 objetivo desse trabalho foi comparar o Método de Dimensionamento Trecho-a-Trecho com o método de múltiplas saídas, com a perda de carga contínua determinada pela Equação de Darcy-Weisbach. Foram feitas simulações de dimensionamento utilizando três tipos de aspersores, variando o número de aspersor por lateral e a pressão de serviço. Em todas as simulações o dimensionamento pelo método de múltiplas saídas apresentou valores superiores ao método trecho-a-trecho, a maior diferença entre os dois métodos chegou a 9,69\% e a menor diferença foi de $0,15 \%$.

Palavras-chave: Perda de carga; Equação de Darcy-Weisbach; Coeficiente de Uniformidade de Christiansen.

Abstract. Comparison between the Step-by-Step Method and the Multiple Output Method in the size of a side line of an sprinkler irrigation system. The design of irrigation systems is essential for its proper functioning and has a direct relation with the consumption of electric energy by the motor pump. The objective of this work is to compare the method of scaling stepby-step with the method of multiple outputs, with the continuous head loss determined by the Darcy-Weisbach Equation. Simulations were performed using three types of sprinklers, varying the number of sprinklers per line and the service pressure. In all the simulations, the multiple-output design showed higher values than the Step-by-Step Method, the biggest difference between the two methods reached $9,69 \%$ and the smallest difference was $0,15 \%$.
Recebido:

18/09/2018

Aceito:

$19 / 12 / 2018$

Publicado:

$31 / 12 / 2018$

Acesso aberto

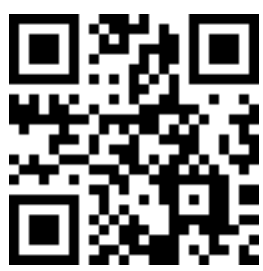

ORCID

(1) 0000-0002-1390-1639

Eugenio Paceli de

Miranda

ㄴ) 0000-0002-1871-7195

Gilbenes Bezerra Rosal 
Keywords: Head loss; Darcy-Weisbach's Equation; Christiansen’s Uniformity Coefficient.

\section{Introdução}

O bom dimensionamento de um sistema de irrigação é imprescindível para economia de água e energia elétrica e para o bom funcionamento dos equipamentos, contribui também para obter boa uniformidade de aplicação.

0 dimensionamento de um sistema de irrigação e seus investimentos podem levar a custos altos, dependendo principalmente de conhecimento técnico, mão de obra e os componentes do sistema, porém considerando-se que o mau dimensionado pode causar baixa uniformidade de aplicação e consequentemente diminuir a produtividade das áreas irrigadas por déficit ou excesso de água, pode-se acarretar ainda mais custos do que um trabalho bem elaborado.

$$
\text { Para Miranda (2016) é }
$$

importante que o dimensionamento leve em consideração também à otimização no uso da energia, exigindo que esse recurso seja usado de forma racional nos aspectos econômico, ambiental e social, já que o mesmo sofre alterações na sua disponibilidade em função das condições climáticas, da matriz energética do país, da crescente demanda, o que leva a eventuais riscos de desabastecimento.

Segundo Rettore Neto (2011) o conhecimento das perdas de carga contínua e localizada ajuda na escolha da bomba e diminui os custos do sistema e de operação do projeto. A perda de carga leva a uma redução da pressão, que reflete diretamente na potência necessária do sistema de bombeamento (Pereira, 2011).

A perda contínua de carga representa a dissipação de energia da água em forma de calor, ao longo da tubulação, decorrente da resistência ao escoamento oferecida pela viscosidade do fluido e pela inércia das partículas, sendo variável com a rugosidade da parede do tubo, diâmetro da tubulação e com a velocidade da água (Porto, 2006).

Coelho et al. (2018) define a perda de carga como uma medida que sofre influência de diversos fatores, dentre eles podem ser destacados a temperatura do fluido, sua viscosidade e a rugosidade da tubulação.

Alguns fatores que influenciam nas perdas de carga são: a natureza do fluido escoado, natureza das paredes dos tubos como o diâmetro e seu envelhecimento, o regime de escoamento do fluido e a velocidade do escoamento (Amaral e Amaral, 2016).

Como a determinação da perda de carga em campo torna-se inviável ao projetista para o dimensionamento hidráulico, logo existem diversas equações que estimam a perda de carga nas tubulações (Coelho et al., 2018).

As equações para estimar a perda de carga foram determinadas ou deduzidas teoricamente para um regime de escoamento específico (Zitterell, 2011). O regime de escoamento pode ser classificado laminar se o Número de Reynolds for até 2.000 e turbulento quando acima de 4.000, valores entre 2.000 e 4.000 são considerados regime de transição, porém em sistemas de irrigação pressurizados geralmente os valores são muito acima de 4.000 .

Como a perda de carga é dependente de diversos fatores, a utilização de equações para sua estimativa é uma tarefa difícil e que muitas vezes não representa a realidade (Alazba et al., 2012).

Geralmente as equações superestimam as perdas de carga que ocorrem, pois as tubulações de polietileno, mais comumente utilizadas em sistemas de irrigação, aumentam seu diâmetro quando pressurizadas, 
diminuindo a perda de carga que é determinada (Rettore Neto et al., 2013).

De acordo com Rettore Neto et al. (2013), a Equação de Darcy-Weisbach, com uso do cálculo do fatos de atrito por Blasius, obtem-se menor variação dos valores comparando com os determinados em laboratório.

Segundo Rettore Neto et al. (2014) é possível minimizar a diferença dos métodos de estimativa da perda de carga, porém necessita de conhecimento especifico dos profissionais pela complexidade e informações muito específicas dos materiais das tubulações como coeficientes de elasticidade, valores que nem sempre são facilmente disponíveis.

No presente trabalho o objetivo foi comparar o Método de Dimensiona- mento Trecho-a-Trecho com o Método de Múltiplas Saídas, foram utilizados três tipos de aspersores variando o número de aspersor por lateral e a pressão de serviço.

\section{Material e métodos}

Determinou-se a perda de carga total da linha lateral de um sistema de irrigação por aspersão através de um programa em linguagem Visual Basic for Aplication (VBA) desenvolvido especialmente para esse trabalho. Foram simulados o dimensionamento com três tipos diferentes de aspersores, cujas equações da vazão estão descritas na Tabela 1. Variou-se as pressões de serviço e o número de aspersores na linha lateral.

Tabela 1. Equação da vazão do aspersor (q - vazão (L.h-1); H - pressão (mca); k - coeficiente matemático; $\mathrm{x}$ - expoente de descarga).

\begin{tabular}{lc}
\hline Emissor & Equação da vazão \\
\hline Aspersor NY-25 (Agropolo), bocal: amareloxcinza & $\mathrm{q}=117,593 \cdot \mathrm{H}^{0,501}$ \\
Aspersor MV-360L (Agropolo), bocal: azulxcinza & $\mathrm{q}=236,967 . \mathrm{H}^{0,499}$ \\
Aspersor NY-30 (Agropolo), bocal: longo vermelho & $\mathrm{q}=643,988 \cdot \mathrm{H}^{0,5}$ \\
\hline
\end{tabular}

A perda de carga contínua foi determinada pela Equação de DarcyWeisbach (Equação 1), para o método trecho-a-trecho e a Equação 2 para o método de múltiplas saídas, com o Fator de Ajuste de Christiansen (Fn), determinado pela Equação 3.

$$
\begin{gathered}
\text { hf }=\mathrm{f} \frac{\mathrm{L}}{\mathrm{D}} \frac{\mathrm{v}^{2}}{2 \mathrm{~g}} \\
\mathrm{hf}=\mathrm{f} \frac{\mathrm{L}}{\mathrm{D}} \frac{\mathrm{v}^{2}}{2 \mathrm{~g}} \cdot F n \\
F n=\frac{1}{m+1}+\frac{1}{2 N}+\frac{\sqrt{m-1}}{6 N^{2}}
\end{gathered}
$$

Em que: hf - Perda de carga (m); f - Fator de atrito (adimensional); L Comprimento da tubulação (m); D Diâmetro interno da tubulação (m); v Velocidade de escoamento ( $\left.\mathrm{m} \mathrm{s}^{-1}\right)$; g Aceleração da gravidade ( $\left.\mathrm{m} \mathrm{s}^{-2}\right)$; Fn Fator de correção de múltiplas saídas; $m$ - expoente da velocidade; $\mathrm{N}$ - número de aspersores.

A perda de carga localizada foi determinada pela equação da energia cinética, aplicando a Equação 4. No método trecho-a-trecho o coeficiente de perda localizada (K) corresponde a uma única peça, o tê de acesso da haste do aspersor. Para o método de múltiplas saídas, a perda de carga localizada foi determinada pela Equação 5. 


$$
\begin{gathered}
\mathrm{hf}_{\mathrm{Loc}}=\mathrm{K} \cdot \frac{\mathrm{v}^{2}}{2 \mathrm{~g}} \\
\mathrm{hf}_{\mathrm{Loc}}=\sum \mathrm{K} \cdot \frac{\mathrm{v}^{2}}{2 \mathrm{~g}} \cdot \mathrm{Fn}
\end{gathered}
$$

Em que: $\mathrm{hf}_{\mathrm{Loc}}$ - Perda localizada de carga (m); K - Coeficiente de perda localizada de carga (adimensional); v Velocidade do fluido $\left(\mathrm{m} \mathrm{s}^{-1}\right) ; \mathrm{g} \quad$ Aceleração da gravidade $\left(\mathrm{m} \mathrm{s}^{2}\right)$.

Para calcular o fator de atrito da equação de Darcy-Weisbach (f) utilizouse a Equação de Hagen-Poiseuille (Equação 6) para regime laminar, Número de Reynolds menor que 2.000 $(\operatorname{Re}<2.000)$ e a Equação de Blasius
(Equação 7), para Números de Reynolds igual ou maior que $2.000(\operatorname{Re} \geq 2.000)$.

$$
\begin{aligned}
& f=\frac{64}{\operatorname{Re}} \\
& f=\frac{0,316}{\operatorname{Re}^{0,25}}
\end{aligned}
$$

Em que: f - Fator de atrito (adimensional); Re - Número de Reynolds (adimensional).

No método trecho-a-trecho (Figura 1) o dimensionamento foi feito a partir do último emissor situado no final da linha lateral, com vazão do último emissor (qn) submetido a uma pressão Hn, conforme Equação 8.
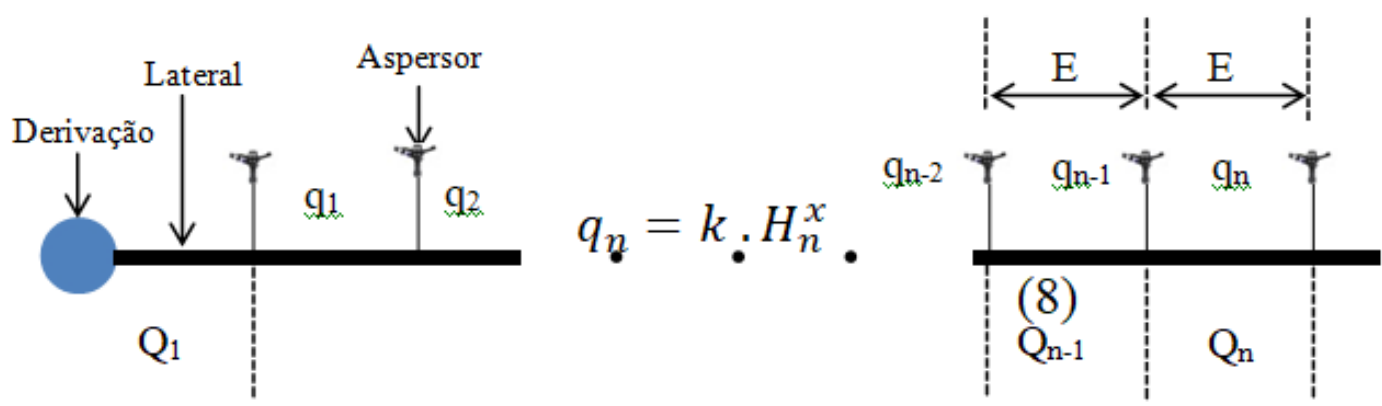

Figura 1. Esquema da disposição dos emissores e dos trechos na linha Lateral $\left(\mathrm{q}_{\mathrm{n}}\right.$ - vazão do último emissor; $\mathrm{q}_{\mathrm{n}-1}$ - vazão do penúltimo emissor; $\mathrm{q}_{\mathrm{n}-2}$ - vazão do antepenúltimo emissor; $\mathrm{q}_{1}$ - vazão do primeiro emissor; $\mathrm{q}_{2}$ - vazão do segundo emissor; $\mathrm{Q}_{\mathrm{n}}$ - vazão do último trecho; $\mathrm{Q}_{\mathrm{n}-1}$ - vazão do penúltimo trecho; $Q_{1}$ - vazão do primeiro trecho; E - espaçamento entre aspersores).

$$
q_{n}=k \cdot H_{n}^{x}
$$

Em que: $\mathrm{q}_{\mathrm{n}}$ - Vazão do último emissor $\left(\mathrm{L} \mathrm{h}^{-1}\right) ; \mathrm{k}$ - Coeficiente de proporcionalidade (adimensional); $\mathrm{H}_{\mathrm{n}}$ Pressão no último emissor (mca); x Expoente de descarga (adimensional).
0 penúltimo emissor possui uma vazão $\left(\mathrm{q}_{\mathrm{n}-1}\right)$ submetida à uma pressão $\left(\mathrm{H}_{\mathrm{n}-1}\right)$ determinada pela Equação 9.

$$
\mathrm{q}_{\mathrm{n}-1}=\mathrm{k} \cdot \mathrm{H}_{\mathrm{n}-1}^{\mathrm{x}}
$$


Em que: $\mathrm{q}_{\mathrm{n}-1}$ - Vazão do penúltimo emissor (L.h $\left.{ }^{-1}\right) ; \mathrm{k}$ - Coeficiente de proporcionalidade (adimensional); $\mathrm{H}_{\mathrm{n}-1}$ Pressão no penúltimo emissor (m); $\mathrm{x}$ Expoente de descarga (adimensional).

Determina-se a pressão do penúltimo emissor $\left(\mathrm{H}_{\mathrm{n}-1}\right)$ pela soma da pressão do último emissor $\left(\mathrm{H}_{\mathrm{n}}\right)$ com a perda de carga no último trecho $\left(\mathrm{Hf}_{\mathrm{n}}\right)$, Equação 10.

$$
\mathrm{H}_{\mathrm{n}-1}=\mathrm{H}_{\mathrm{n}}+\mathrm{Hf}_{\mathrm{n}}
$$

Em que: $\mathrm{H}_{\mathrm{n}-1}$ - Pressão do penúltimo emissor (mca); $\mathrm{H}_{\mathrm{n}}$ - Pressão do último emissor (mca); $\mathrm{Hf}_{\mathrm{n}}$ - Perda de carga do último trecho $(\mathrm{m})$.

A vazão no penúltimo trecho $\left(Q_{n-1}\right)$ corresponde a soma das vazões do último $\left(\mathrm{q}_{\mathrm{n}}\right)$ e do penúltimo emissor $\left(\mathrm{q}_{\mathrm{n}-1}\right)$, Equação 11.

$$
\mathrm{Q}_{\mathrm{n}-1}=q_{\mathrm{n}-1}+q_{n}
$$

Em que: $\mathrm{Q}_{\mathrm{n}-1}$ - Vazão do penúltimo trecho $\left(\mathrm{L} \mathrm{h}^{-1}\right) ; \mathrm{q}_{\mathrm{n}}$ - Vazão do último emissor $\left(L^{h}{ }^{-1}\right) ; q_{n-1}$ - Vazão do penúltimo emissor $\left(\mathrm{L} \mathrm{h}^{-1}\right)$;

A pressão de um determinado aspersor vai ser sempre a perda de carga do trecho imediatamente posterior a ele mais a pressão do aspersor seguinte a esse trecho, fazendo assim, sucessivamente, até o início da linha lateral (Figura 2).

Os parâmetros hidráulicos usados para a simulação do dimensionamento da linha lateral são mostrados na Tabela 2 .

$$
\mathrm{H}_{\mathrm{n}-2}=\mathrm{H}_{\mathrm{n}-1}+\mathrm{Hf}_{\mathrm{n}-1}
$$$$
\mathrm{H}_{\mathrm{n}-1}=\mathrm{H}_{\mathrm{n}}+\mathrm{Hf}_{\mathrm{n}}
$$$$
\mathrm{H}_{\mathrm{n}}=\mathrm{PS}
$$

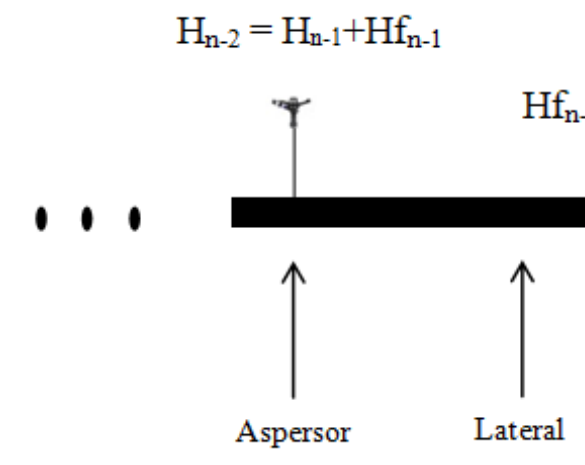

Figura 2. Esquema da determinação das pressões em cada emissor $\left(H_{n}\right.$ - pressão do último aspersor; $\mathrm{H}_{\mathrm{n}-1}$ - pressão do penúltimo aspersor; $\mathrm{H}_{\mathrm{n}-2}$ - pressão do antepenúltimo aspersor; $\mathrm{Hf}_{\mathrm{n}}$ perda de carga no último trecho; $\mathrm{Hf}_{\mathrm{n}-1}$ - perda de carga no penúltimo trecho; PS - pressão de serviço).

Tabela 2. Parâmetros hidráulicos para dimensionamento da linha lateral.

\begin{tabular}{lccc}
\hline \multirow{2}{*}{ Parâmetros } & \multicolumn{3}{c}{ Aspersores } \\
\cline { 2 - 4 } & NY-25 & MV-360L & NY-30 \\
\hline Bocal & amareloxcinza & azulxcinza & Longo vermelho \\
Diâmetro do bocal (mm) & $2,5 \times 2,5$ & $3,5 \times 2,5$ & $6,2 \times 4,5$ \\
Diâmetro da Linha Lateral (mm) & 50 & 75 & 75 \\
Espaçamento entre aspersores (m) & 6,0 & 6,0 & 12,0 \\
Coeficiente K de perda de carga localizada & 1,8 & 1,8 & 1,8 \\
\hline
\end{tabular}




\section{Resultados e discussão}

Nas Figuras 3, 4 e 5 são mostradas as perdas de cargas totais, contínua mais localizada, para o método trecho-a-trecho (Hf t-t) e o método de múltiplas saídas (Hf ms) em função do aumento do número de emissores.

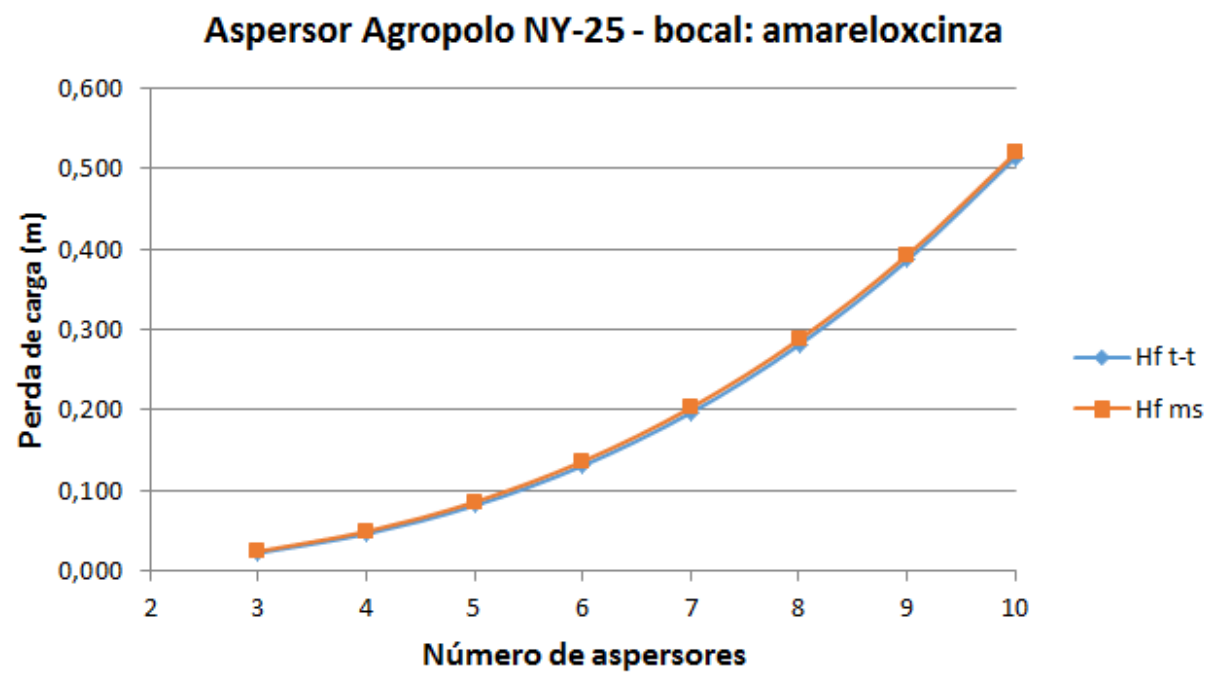

Figura 3. Perda de carga total para o aspersor NY-25 (bocal: amarelo x cinza) em função do número de aspersores.

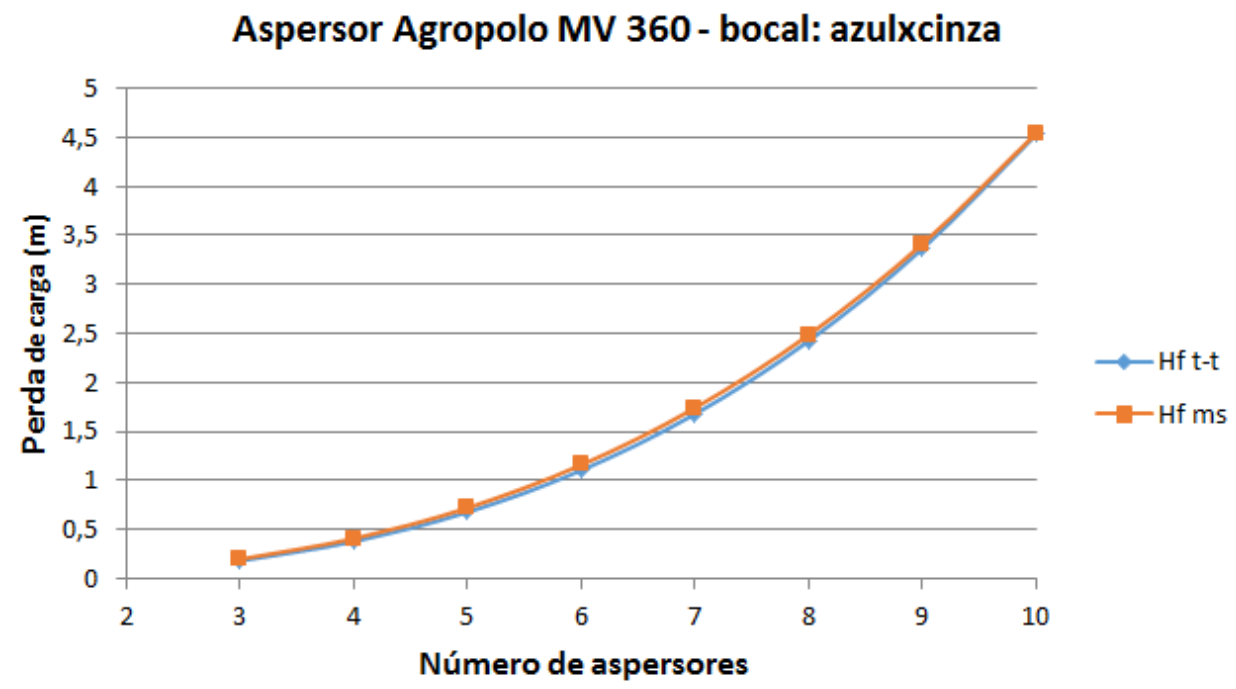

Figura 4. Perda de carga total para o aspersor MV-360L (bocal: azul x cinza) em função do número de aspersores. 


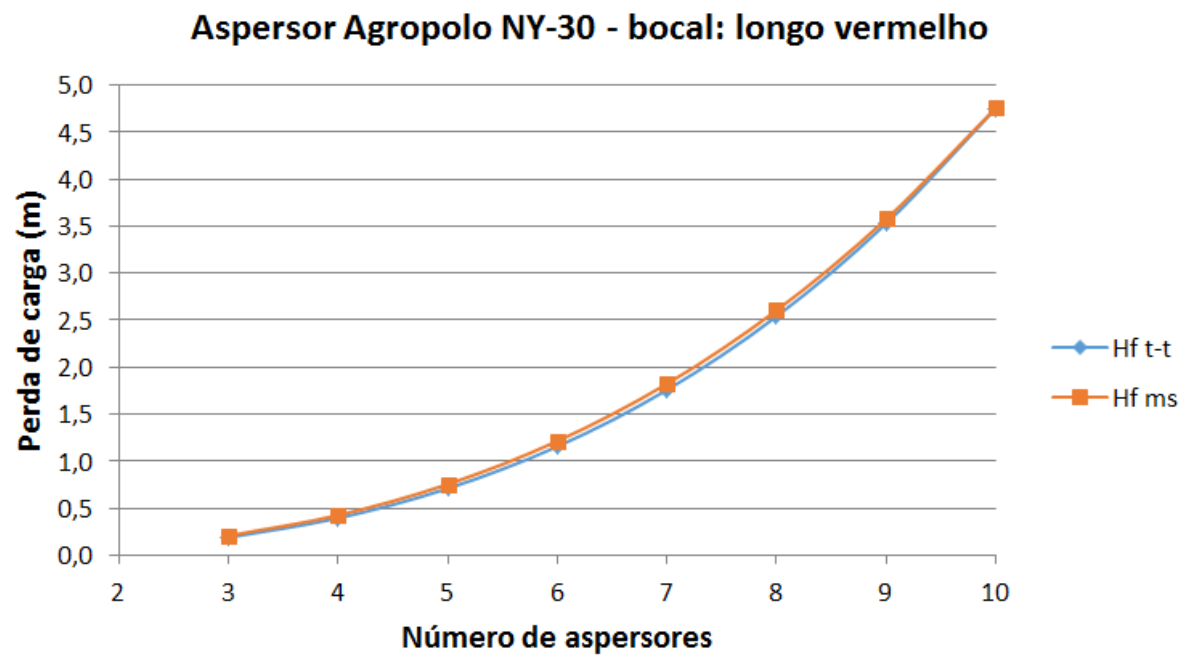

Figura 5. Perda de carga total para o aspersor NY-30 (bocal: longo vermelho) em função do número de aspersores.

Observa-se que as perdas de cargas apresentadas pelos dois métodos são praticamente iguais. Pela Tabela 3 é possível observar que as perdas de cargas determinadas pelo método de múltiplas saídas são um pouco maiores que o método trecho-a-trecho, a maior diferença entre os dois métodos foi de $9,69 \%$, em média a diferença entre os dois métodos foi de $4,44 \%$.

Tabela 3. Perda de carga total em função do número de aspersores e diferenças entre o método trecho-a-trecho e de múltiplas saídas.

\begin{tabular}{cccccccccc}
\hline \multirow{2}{*}{ № asp. } & \multicolumn{3}{c}{ NY-25 } & \multicolumn{3}{c}{ MY-360L } & \multicolumn{3}{c}{ NY-30 } \\
\cline { 2 - 10 } & Hf t-t & Hf $\mathbf{~ m s}$ & $\boldsymbol{\Delta} \mathbf{( \% )}$ & Hf t-t & Hf $\mathbf{~ m s}$ & $\boldsymbol{\Delta} \mathbf{( \% )}$ & Hf t-t & Hf ms & $\boldsymbol{\Delta} \mathbf{( \% )}$ \\
\hline 3 & 0,022 & 0,024 & $-8,65$ & 0,182 & 0,199 & $-9,69$ & 0,190 & 0,209 & $-9,69$ \\
4 & 0,046 & 0,049 & $-6,82$ & 0,378 & 0,408 & $-7,91$ & 0,396 & 0,427 & $-7,91$ \\
5 & 0,081 & 0,086 & $-5,32$ & 0,679 & 0,722 & $-6,35$ & 0,711 & 0,756 & $-6,34$ \\
6 & 0,131 & 0,136 & $-4,12$ & 1,105 & 1,160 & $-4,98$ & 1,157 & 1,215 & $-4,96$ \\
7 & 0,197 & 0,203 & $-3,14$ & 1,678 & 1,741 & $-3,74$ & 1,759 & 1,824 & $-3,70$ \\
8 & 0,281 & 0,288 & $-2,32$ & 2,424 & 2,486 & $-2,57$ & 2,540 & 2,604 & $-2,51$ \\
9 & 0,386 & 0,392 & $-1,63$ & 3,365 & 3,413 & $-1,43$ & 3,528 & 3,575 & $-1,34$ \\
10 & 0,514 & 0,519 & $-1,03$ & 4,528 & 4,540 & $-0,27$ & 4,749 & 4,756 & $-0,15$ \\
\hline
\end{tabular}

Nas Figuras 6, 7 e 8 são mostradas as perdas de carga total determinadas pelos dois métodos, variando a pressão de serviço para uma linha lateral dimensionada com 10 aspersores. 


\section{Aspersor Agropolo NY-25 - bocal: amareloxcinza}

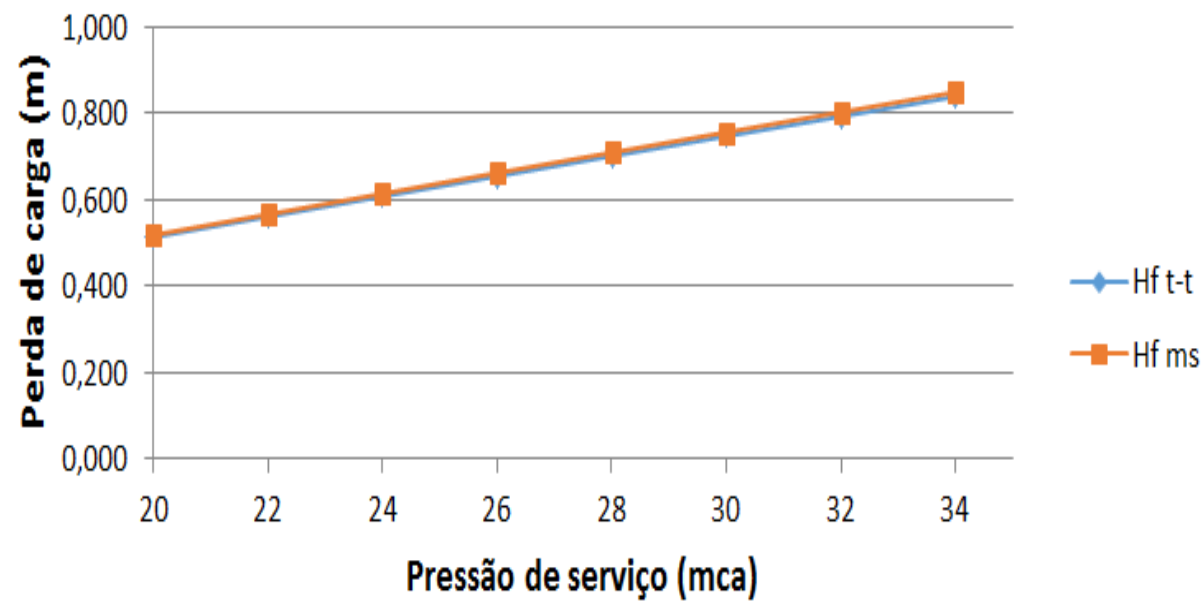

Figura 6. Perda de carga total do aspersor Agropolo NY-25 (bocal: amarelo x cinza) em função da pressão de serviço do aspersor

\section{Aspersor Agropolo MV 360 - bocal: azulxcinza}

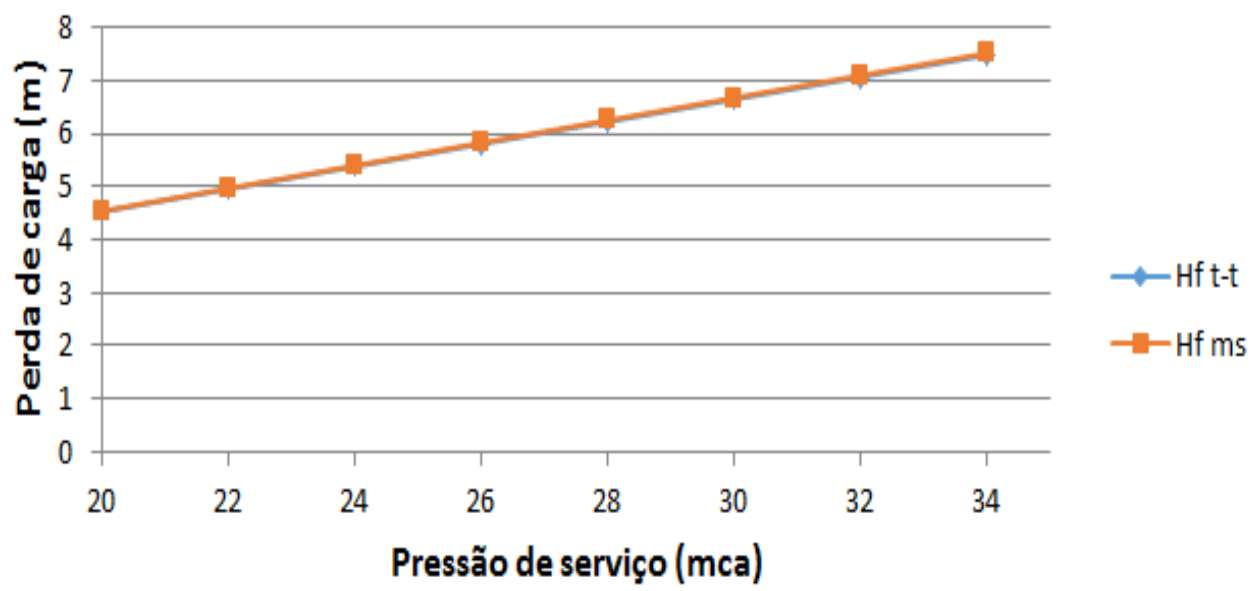

Figura 7. Perda de carga total do aspersor Agropolo MV-360L (bocal: azul x cinza) em função da pressão de serviço do aspersor 


\section{Aspersor Agropolo NY-30 - bocal: longo vermelho}

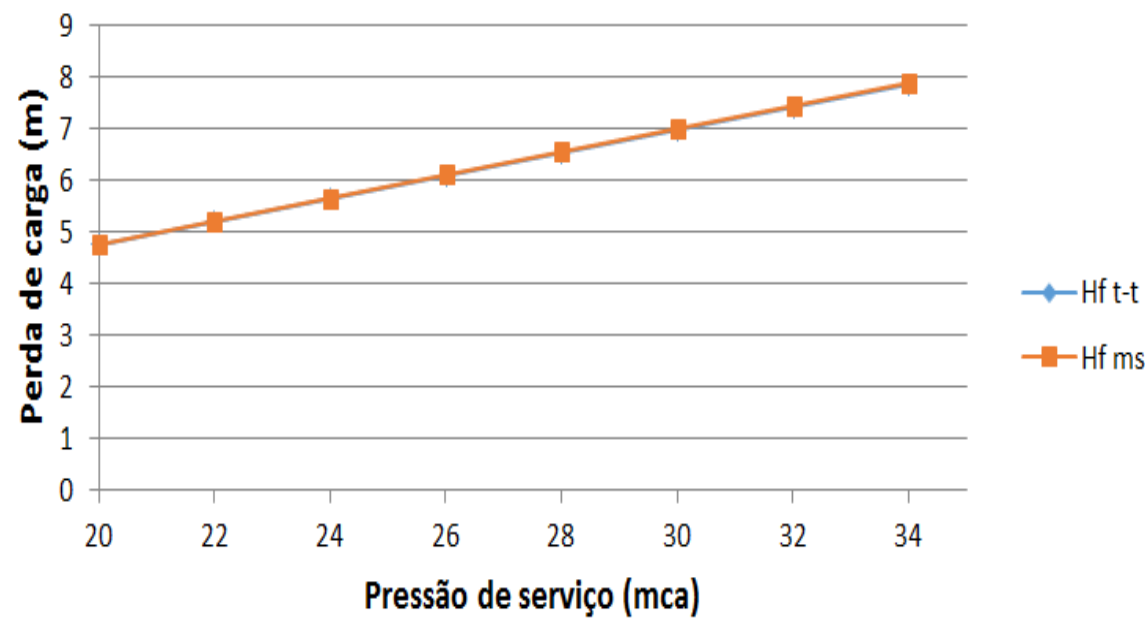

Figura 8. Perda de carga total do aspersor Agropolo NY-30 (bocal: longo vermelho) em função da pressão de serviço do aspersor.

O comportamento da perda de carga total em função da pressão é praticamente igual em relação aos dois métodos de determinação. Na Tabela 4 é possível verificar essas diferenças. Aqui também a perda de carga determinada pelo método de múltiplas saídas também foi ligeiramente maior. A maior diferença encontrada foi de 1,26\%, em média a diferença foi de $0,58 \%$.

Tabela 4. Perda de carga total em função da pressão de serviço.

\begin{tabular}{cccccccccr}
\hline $\mathbf{P}$ & \multicolumn{3}{c}{$\mathbf{N Y - 2 5}$} & \multicolumn{3}{c}{ MY-360L } & \multicolumn{3}{c}{ NY-30 } \\
\cline { 2 - 10 } (mca) & Hf t-t & Hf ms & $\boldsymbol{\Delta} \mathbf{( \% )}$ & Hf t-t & Hf $\mathbf{~ m s}$ & $\boldsymbol{\Delta} \mathbf{( \% )}$ & Hf t-t & Hf ms & $\Delta(\mathbf{m})$ \\
\hline 20 & 0,514 & 0,519 & $-0,97$ & 4,528 & 4,540 & $-0,27$ & 4,749 & 4,756 & $-0,15$ \\
22 & 0,561 & 0,567 & $-1,00$ & 4,955 & 4,970 & $-0,30$ & 5,198 & 5,208 & $-0,19$ \\
24 & 0,609 & 0,615 & $-1,07$ & 5,380 & 5,398 & $-0,33$ & 5,645 & 5,657 & $-0,22$ \\
26 & 0,655 & 0,663 & $-1,18$ & 5,803 & 5,824 & $-0,36$ & 6,090 & 6,105 & $-0,25$ \\
28 & 0,702 & 0,710 & $-1,14$ & 6,225 & 6,249 & $-0,39$ & 6,534 & 6,552 & $-0,27$ \\
30 & 0,748 & 0,757 & $-1,20$ & 6,645 & 6,673 & $-0,41$ & 6,976 & 6,997 & $-0,30$ \\
32 & 0,794 & 0,804 & $-1,26$ & 7,064 & 7,095 & $-0,44$ & 7,417 & 7,441 & $-0,32$ \\
34 & 0,840 & 0,850 & $-1,19$ & 7,482 & 7,516 & $-0,46$ & 7,856 & 7,883 & $-0,34$ \\
\hline
\end{tabular}

As perdas de carga contínua e localizada foram analisadas separadamente em função do número de aspersores. Nas Figuras 9, 10 e 11 e na Tabela 5 são mostradas as perdas de carga contínua determinadas por cada método e nas Figuras 12, 13 e 14 e na Tabela 6 são mostradas as perdas de carga localizada. 


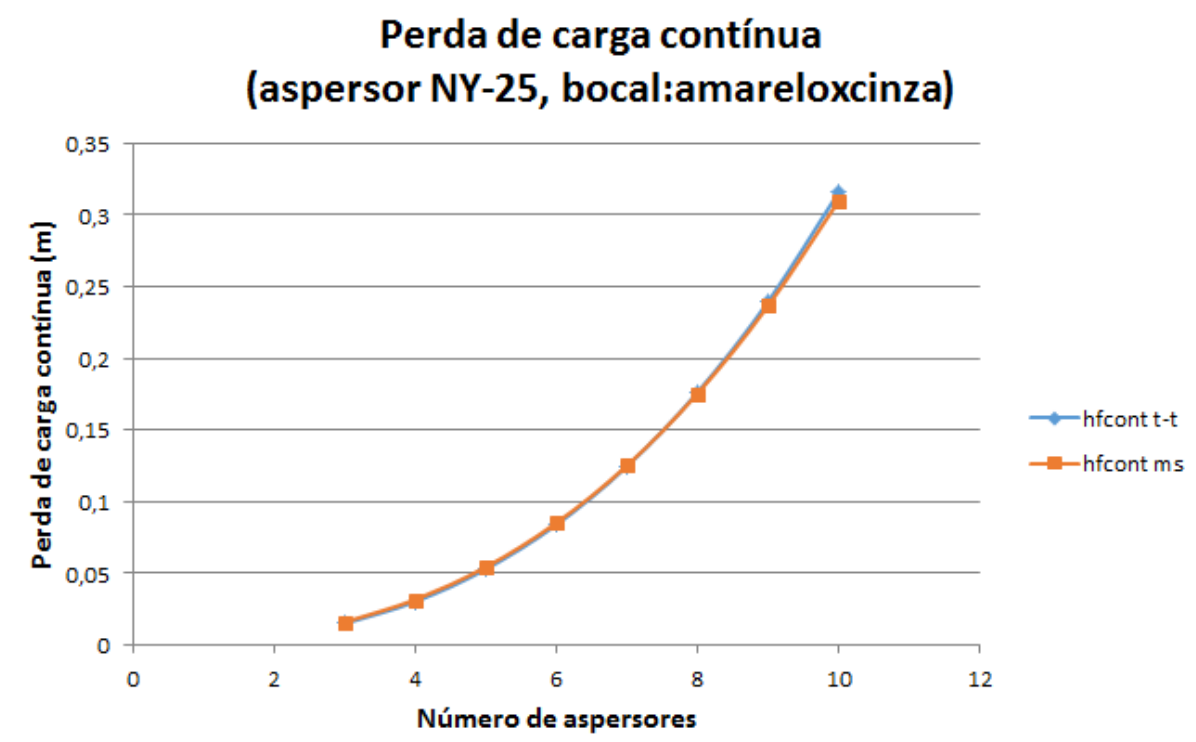

Figura 9. Perda de carga contínua do aspersor Agropolo NY-25 (bocal: amarelo x cinza) em função do número de aspersores.

\section{Aspersor Agropolo NY-30 - bocal: longo vermelho}

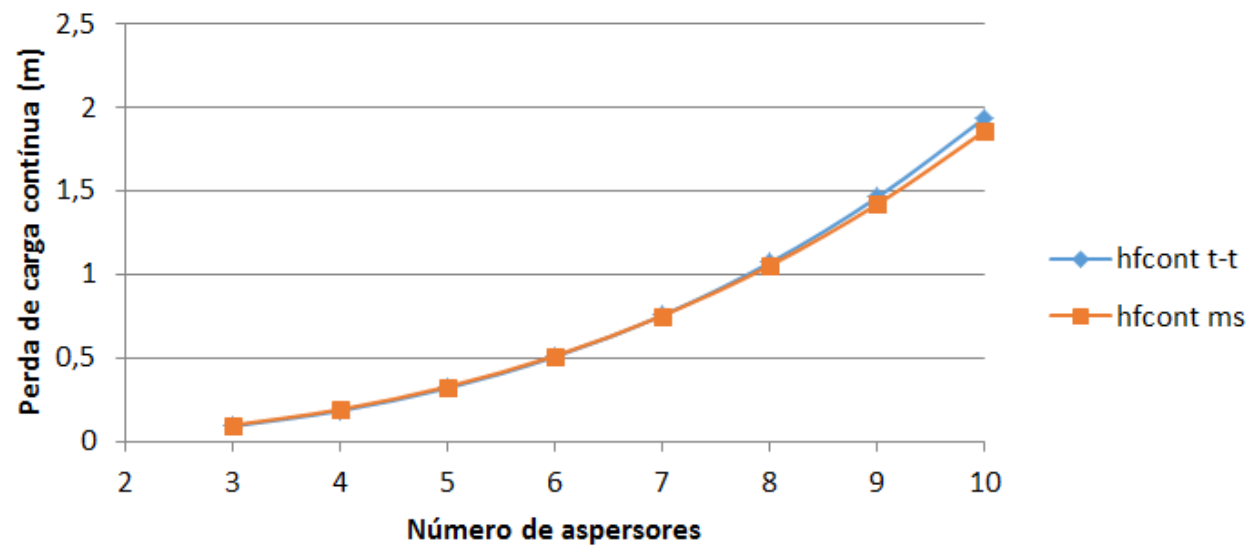

Figura 10. Perda de carga contínua do aspersor Agropolo NY-30 (bocal: longo vermelho) em função do número de aspersores. 


\section{Aspersor Agropolo MV 360 - bocal: azulxcinza}

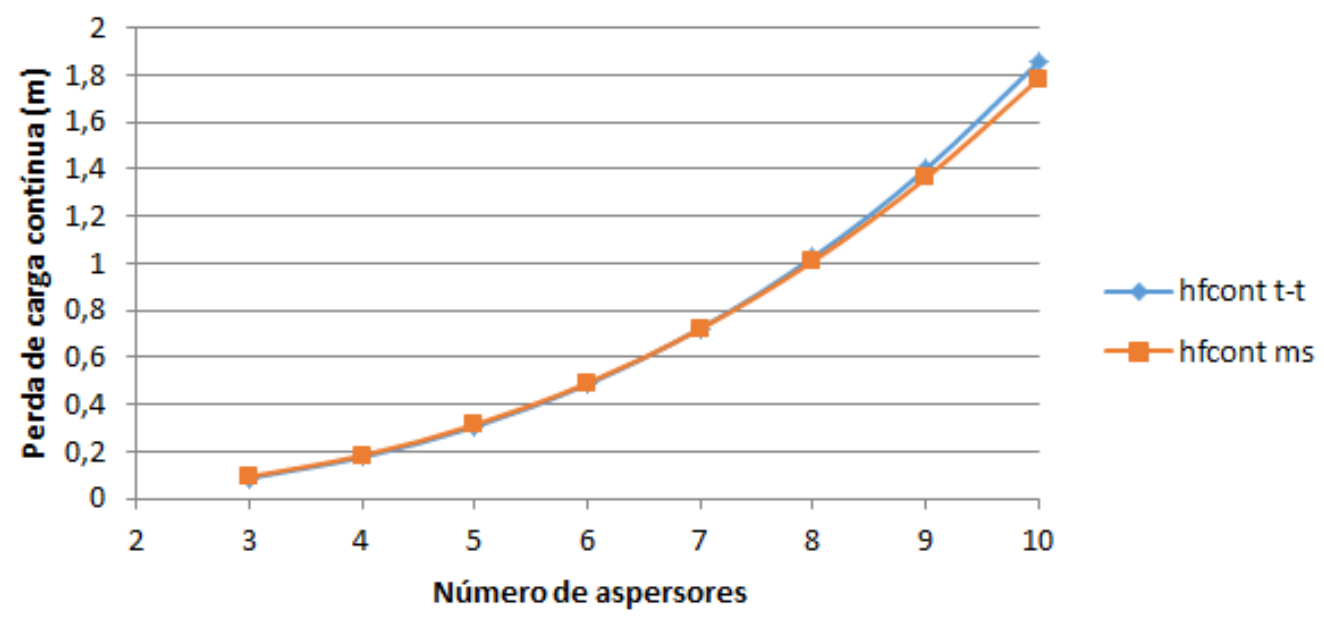

Figura 11. Perda de carga contínua do aspersor Agropolo MV 360 (bocal: azul x cinza) em função do número de aspersores.

Tabela 5. Perdas de carga contínua em função do número de aspersores.

\begin{tabular}{cccccccccr}
\hline $\mathbf{P}$ & \multicolumn{3}{c}{$\mathbf{N Y - 2 5}$} & \multicolumn{3}{c}{ MY-360L } & \multicolumn{3}{c}{ NY-30 } \\
\cline { 2 - 10 } (mca) & Hf t-t & Hf $\mathbf{~ m s}$ & $\boldsymbol{\Delta} \mathbf{( \% )}$ & Hf t-t & Hf ms & $\boldsymbol{\Delta} \mathbf{( \% )}$ & Hf t-t & Hf $\mathbf{~ m s}$ & $\boldsymbol{\Delta} \mathbf{( \% )}$ \\
\hline 3 & 0,015 & 0,016 & $-6,82$ & 0,087 & 0,093 & $-6,74$ & 0,091 & 0,097 & $-6,74$ \\
4 & 0,030 & 0,032 & $-4,66$ & 0,175 & 0,183 & $-4,49$ & 0,183 & 0,191 & $-4,48$ \\
5 & 0,053 & 0,055 & $-2,94$ & 0,305 & 0,313 & $-2,63$ & 0,319 & 0,327 & $-2,61$ \\
6 & 0,084 & 0,085 & $-1,57$ & 0,486 & 0,491 & $-1,06$ & 0,507 & 0,512 & $-1,03$ \\
7 & 0,125 & 0,125 & $-0,46$ & 0,723 & 0,721 & 0,31 & 0,755 & 0,752 & 0,35 \\
8 & 0,176 & 0,175 & 0,46 & 1,024 & 1,009 & 1,55 & 1,070 & 1,053 & 1,61 \\
9 & 0,240 & 0,237 & 1,23 & 1,399 & 1,360 & 2,73 & 1,461 & 1,420 & 2,81 \\
10 & 0,316 & 0,310 & 1,90 & 1,853 & 1,781 & 3,89 & 1,937 & 1,859 & 4,00 \\
\hline
\end{tabular}

No aspersor NY-25 a perda de carga contínua determinada pelo método trecho-a-trecho foi menor até uma linha lateral com 7 aspersores, a maior diferença foi para uma linha lateral com três aspersores, onde a perda de carga contínua chegou próximo de $7 \mathrm{~m}$, diferença que foi diminuindo até uma linha lateral com 7 aspersores. Para esse aspersor a perda de carga contínua, determinada pelo método de múltiplas saídas, aumentou a partir de uma linha lateral com 8 aspersores.

Nos aspersores MY-360L e NY-30, o comportamento da perda de carga contínua foi muito parecido, sendo que até um número de seis aspersores a perda de carga contínua determinada pelo método trecho-a-trecho foi menor. Para esses aspersores a maior diferença foi de $6,74 \%$. 


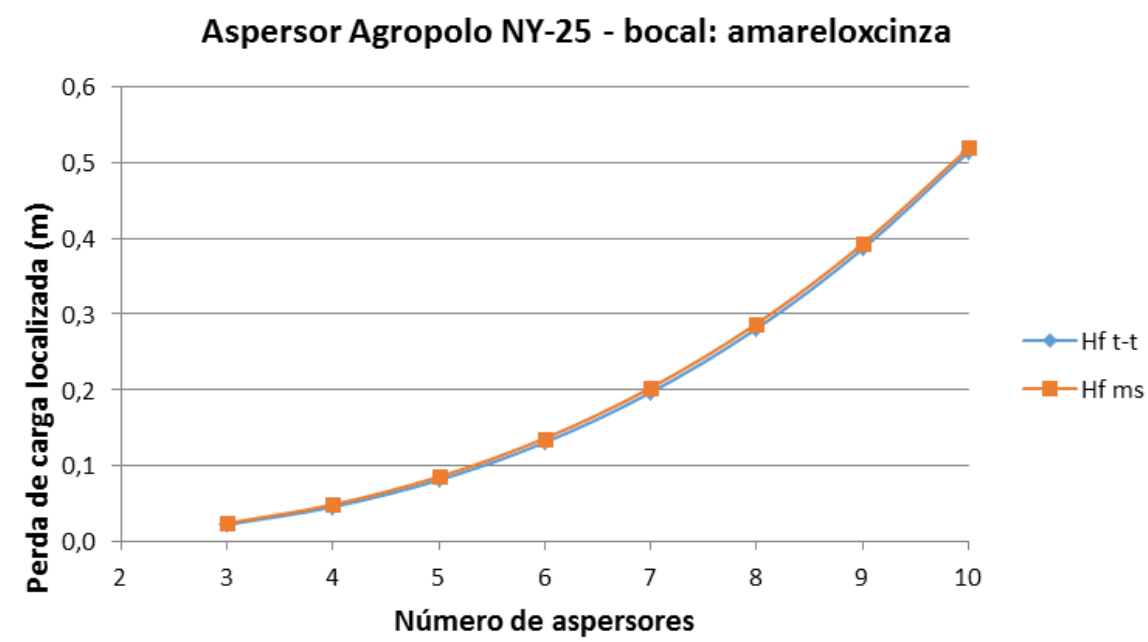

Figura 12. Perda de carga localizada do aspersor Agropolo NY-25 (bocal: amarelo x cinza) em função do número de aspersores.

Aspersor Agropolo NY-30 - bocal: longo vermelho

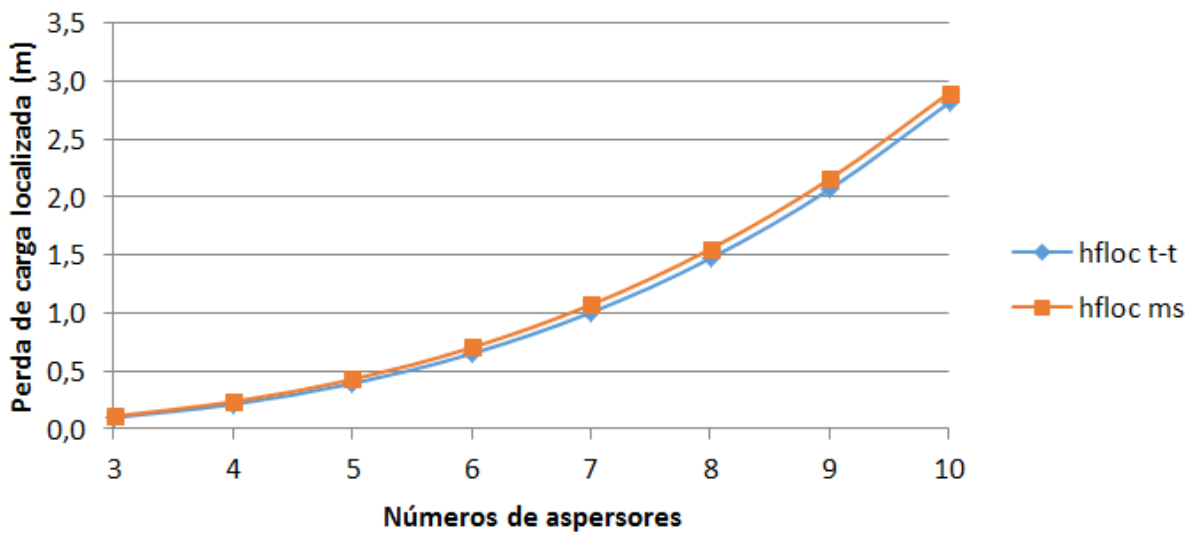

Figura 13. Perda de carga localizada do aspersor Agropolo NY-30 (bocal: longo vermelho) em função do número de aspersores. 
Aspersor Agropolo MV 360 - bocal: azulxcinza

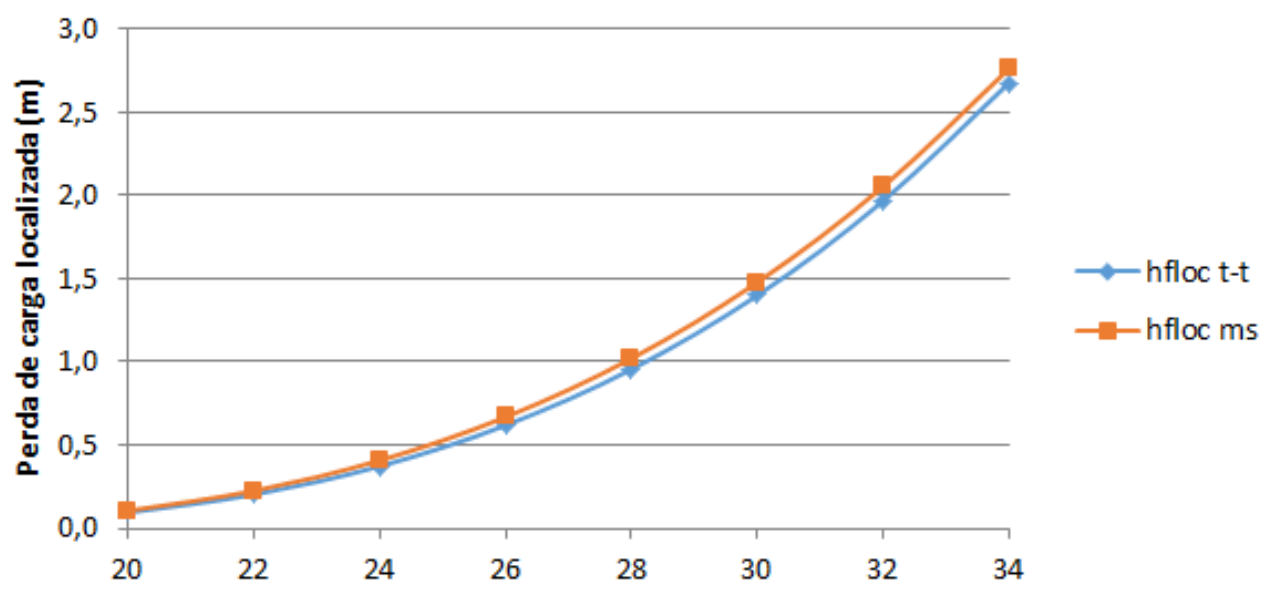

Figura 14. Perda de carga localizada do aspersor Agropolo MV 360 (bocal: azul x cinza) em função do número de aspersores.

Tabela 6. Perdas de carga localizadas em função do número de aspersores.

\begin{tabular}{|c|c|c|c|c|c|c|c|c|c|}
\hline \multirow{2}{*}{$\begin{array}{c}P \\
\text { (mca) }\end{array}$} & \multicolumn{3}{|c|}{ NY-25 } & \multicolumn{3}{|c|}{ MY-360L } & \multicolumn{3}{|c|}{ NY-30 } \\
\hline & Hf t-t & Hf ms & $\Delta(\%)$ & Hf t-t & Hf ms & $\Delta(\%)$ & Hf t-t & Hf ms & $\Delta(\%)$ \\
\hline 3 & 0,007 & 0,008 & $-12,5$ & 0,095 & 0,106 & $-12,39$ & 0,099 & 0,112 & $-12,38$ \\
\hline 4 & 0,015 & 0,017 & $-11,1$ & 0,203 & 0,225 & $-10,87$ & 0,213 & 0,237 & $-10,85$ \\
\hline 5 & 0,028 & 0,031 & $-9,8$ & 0,373 & 0,408 & $-9,40$ & 0,392 & 0,429 & $-9,38$ \\
\hline 6 & 0,047 & 0,051 & $-8,7$ & 0,619 & 0,669 & $-8,05$ & 0,650 & 0,702 & $-8,02$ \\
\hline 7 & 0,072 & 0,077 & $-7,8$ & 0,956 & 1,021 & $-6,80$ & 1,004 & 1,072 & $-6,75$ \\
\hline 8 & 0,105 & 0,112 & $-7,0$ & 1,399 & 1,477 & $-5,59$ & 1,470 & 1,552 & $-5,51$ \\
\hline 9 & 0,147 & 0,156 & $-6,3$ & 1,966 & 2,052 & $-4,39$ & 2,067 & 2,155 & $-4,28$ \\
\hline 10 & 0,198 & 0,209 & $-5,7$ & 2,675 & 2,759 & $-3,15$ & 2,813 & 2,897 & $-3,01$ \\
\hline
\end{tabular}

Como pode ser observado nas Figuras 12,13 e 14 e na Tabela 6 , as diferenças na determinação da perda de carga localizada entre os dois métodos diminuíram com o aumento do número de aspersores, a maior diferença observada foi de $12,5 \%$.
Na Figura 15 são mostradas as perdas de carga total (Hf), contínua (hfcont) e localizada (hfloc) para o aspersor Agropolo NY-25 (bocal: amareloxcinza) em função do número de aspersores pelo método trecho-a-trecho e na Tabela 7 são mostrados os valores das perdas de carga. 


\section{Perda de carga total, contínua e localizada (aspersor NY-25, bocal:amareloxcinza)}

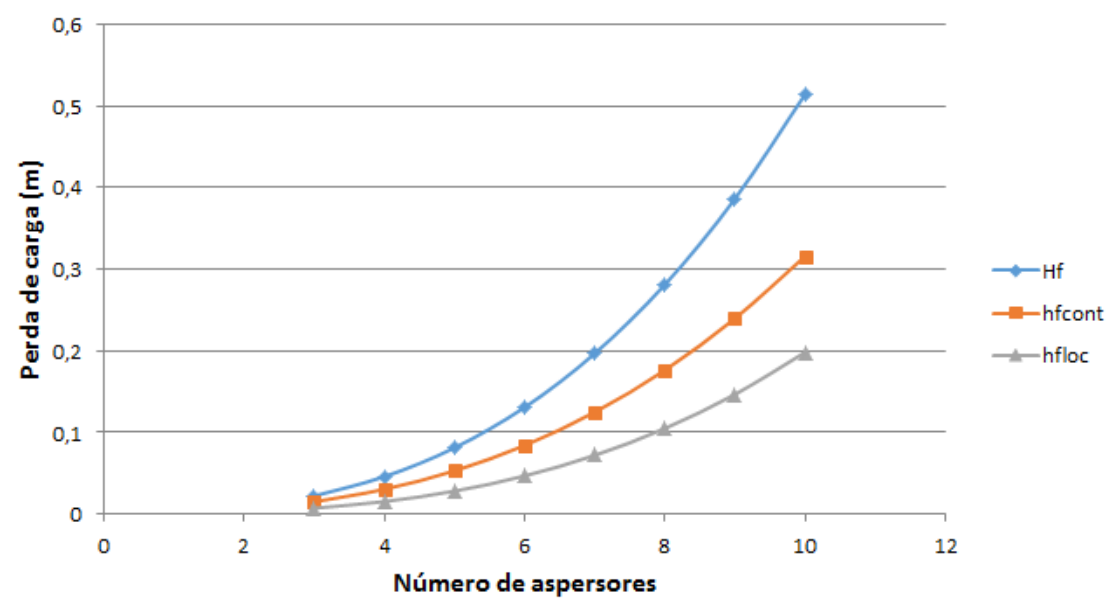

Figura 15. Perda de carga total (Hf), contínua (hfcont) e localizada (hfloc) do aspersor Agropolo NY-25 (bocal: amarelo x cinza) em função do número de aspersores.

Tabela 7. Perdas de carga total (Hf), contínua (hfcont) e localizada (hfloc) do aspersor Agropolo NY-25 (bocal: amarelo x cinza) em função do número de aspersores.

\begin{tabular}{cccccc}
\hline № de asp. & Hf & hfcont & hfloc & $\boldsymbol{\Delta}$ hfcont (\%) & $\boldsymbol{\Delta}$ hfloc (\%) \\
\hline 3 & 0,0223 & 0,0151 & 0,0072 & 67,8 & 32,2 \\
4 & 0,0458 & 0,0304 & 0,0154 & 66,4 & 33,6 \\
5 & 0,0812 & 0,0530 & 0,0282 & 65,2 & 34,8 \\
6 & 0,1308 & 0,0840 & 0,0467 & 64,3 & 35,7 \\
7 & 0,1967 & 0,1248 & 0,0719 & 63,4 & 36,6 \\
8 & 0,2811 & 0,1762 & 0,1048 & 62,7 & 37,3 \\
9 & 0,3861 & 0,2396 & 0,1466 & 62,0 & 38,0 \\
10 & 0,5140 & 0,3158 & 0,1981 & 61,4 & 38,6 \\
\hline
\end{tabular}

É possível verificar que a perda de carga contínua foi sempre superior a perda de carga localizada, em média a perda de carga contínua representou em torno de $63 \%$ da perda de carga total.

\section{Conclusões}

De modo geral, as perdas de carga determinadas pelo método de múltiplas saídas foram maiores que aquela determinadas pelo método trecho-atrecho. A diferença diminui com o aumento do número de aspersores e aumentou levemente com o aumento da pressão de serviço.

\section{Conflito de interesses}

Os autores declaram não haver conflito de interesses.

\section{Referências}

Alazba, A. A.; Mattar, M. A.; Elnesr, M. N.; Amin, M. T. Field assessment of friction head loss and friction correction factor equations. Journal of Irrigation and Drainage Engineering, v. 138, n. 2, p. 166-176, 2012. https://doi.org/10.1061/(ASCE)IR.19434774.0000387

Amaral, E. R.; Amaral, T. R. Análise dos fatores que influenciam nas perdas de carga 
em tubulações e acessórios hidráulicos. Anais do Seminário se Iniciação Cientifica, IFNMG, Montes Claros, 2016.

Coelho, A. P; Zanini, J. R; Faria, R. T. De; Dalri, A. B; Palaretti, L. F. Comparação de equações para estimativa da perda de carga em tubulação de polietileno. Pesquisa Aplicada \& Agrotecnologia, v. 11, n. 1, p. 25-31, 2018. https://doi.org/10.5935/PAeT.V11.N1.03

Miranda E. P. Programa computacional para dimensionamento e determinação dos custos de energia elétrica de um sistema de irrigação localizada. Botucatu: Universidade Estadual Paulista "Júlio de Mesquita Filho" Faculdade De Ciências Agronômicas, 2016. (Tese de doutorado).

Pereira, F. N. Determinação experimental da curva do sistema e da potência consumida em unidade de bombeamento alimentando dois tanques em desnível com fluidos diferentes. Campinas: Universidade Estadual de Campinas, Faculdade de Engenharia Química, 2011. (Dissertação de mestrado).

Porto, R. M. Hidráulica básica. 4. ed. São Carlos: EESC-USP, 2006.
Rettore Neto, O. Modelo para determinação da perda de carga contínua em tubos elásticos. Piracicaba: Universidade de São Paulo, Escola Superior de Agricultura "Luis de Queiroz", 2011. (Tese de doutorado).

Rettore Neto, O.; Botrel, T. A.; Frizzone, J. A.; Pinto, M. F.; Camargo, A. P. Quantificação do erro na determinação da perda contínua de carga em tubos elásticos. Engenharia Agrícola, v. 33, n. 6, p. 1312-1321, 2013. https://doi.org/10.1590/S0100-691620130 00600023

Rettore Neto, O.; Botrel, T. A.; Frizzone, J. A.; Camargo, A.P. Method for determining friction head loss along elastic pipes. Irrigation Science, v. 32, p. 329-339, 2014. https://doi.org/10.1007/s00271-014-04317

Zitterell, D. B. Perda localizada de carga em conectores utilizados em microirrigação. Piracicaba: Universidade de São Paulo, Escola Superior de Agricultura "Luis de Queiroz", 2011. (Dissertação de mestrado).

Informação da Licença: Este é um artigo Open Access distribuído sob os termos da Licença Creative Commons Attribution, que permite uso irrestrito, distribuição e reprodução em qualquer meio, desde que a obra original seja devidamente citada. 